\title{
Paper-based detection device for chronic wound monitoring
}

\author{
Ting Yang ${ }^{1}$, Shin-Chen Pan ${ }^{2,3}$, Chao-Min Cheng ${ }^{4}$ \\ ${ }^{1}$ Department of Chemistry, National Tsing Hua University, Hsinchu, Taiwan; ${ }^{2}$ Division of Plastic and Reconstructive Surgery, Department of \\ Surgery, National Cheng Kung University Hospital, College of Medicine, National Cheng Kung University, Tainan, Taiwan; ${ }^{3}$ International Center \\ for Wound Repair and Regeneration, National Cheng Kung University, Tainan, Taiwan; ${ }^{4}$ Institute of Biomedical Engineering, National Tsing Hua \\ University, Hsinchu, Taiwan \\ Contributions: (I) Conception and design: All authors; (II) Administrative support: All authors; (III) Provision of study material or patients: All authors; \\ (IV) Collection and assembly of data: All authors; (V) Data analysis and interpretation: All authors; (VI) Manuscript writing: All authors; (VII) Final \\ approval of manuscript: All authors. \\ Correspondence to: Chao-Min Cheng. Institute of Biomedical Engineering, National Tsing Hua University, No.101, Sec. 2, Guangfu Rd., East Dist., \\ Hsinchu 300, Taiwan. Email: chaomin@mx.nthu.edu.tw.
}

\begin{abstract}
Background: Chronic wounds are wounds that are impeded at a certain recovery stage and cannot proceed through a normal healing process. They account for an annual wound care expenditure of approximately 5-10 billion dollars. Among all potential patients, the elderly are the most vulnerable population for chronic wounds. Older patients, however, present several issues that must be addressed in order to provide suitable chronic wound diagnosis and care monitoring. Here, we discuss our development of a paper-based detection device that can serve as a rapid, inexpensive, convenient, and sensitive chronic wound assessment tool for providing better chronic wound-monitoring platform among the elderly.

Methods: Our paper-based detection device primarily comprises two parts: a test zone made of chromatography paper, and a passage zone made of Whatman filter paper. In this design, our targeted protein, human neutrophil elastase (HNE), reacts with a pre-impregnated substrate to produce a visible and measurable colorimetric reaction (from colorless to light yellow). An image of the results can be captured with a smart phone camera, and converted to the blue color range using ImageJ software to quantify the mean HNE intensity, defined as test zone intensity minus blank zone intensity.

Results: Our tool produced colorimetric responses using samples from a total of 8 patients, and mean HNE intensities were calculated.

Conclusions: Our paper-based device is designed to be a user-friendly, inexpensive, rapid, and accurate chronic wound assessment platform that can be applied to elderly healthcare and long-term care at home. It can provide for better healthcare outcomes via early chronic wound detection, decreased medical costs, and reduced clinical visit time, all of which would be particularly impactful for the elderly.
\end{abstract}

Keywords: Chronic wounds assessment; paper-based diagnostics; human neutrophil elastase (HNE)

Received: 25 January 2020; Accepted: 31 July 2020; Published: 11 August 2020.

doi: $10.21037 /$ ht-20-6

View this article at: http://dx.doi.org/10.21037/ht-20-6

\section{Introduction}

Chronic wounds are wounds that are impeded at a certain recovery stage and have failed to proceed through a normal healing process within 3 months. Annual costs from chronic wound care are approximately 5 billion to 10 billion dollars $(1,2)$. According to the Wound Healing Society, chronic wounds can be classified into four categories: diabetic ulcers, venous ulcers, pressure ulcers, and arterial insufficiency ulcers (3). During the long recovery time for chronic wounds, patients may undergo emotional or physical trauma, severe pain, a sense of social isolation, and reduced mobility (4). This may ultimately lead to additional, escalated adverse events including infection, amputation, 


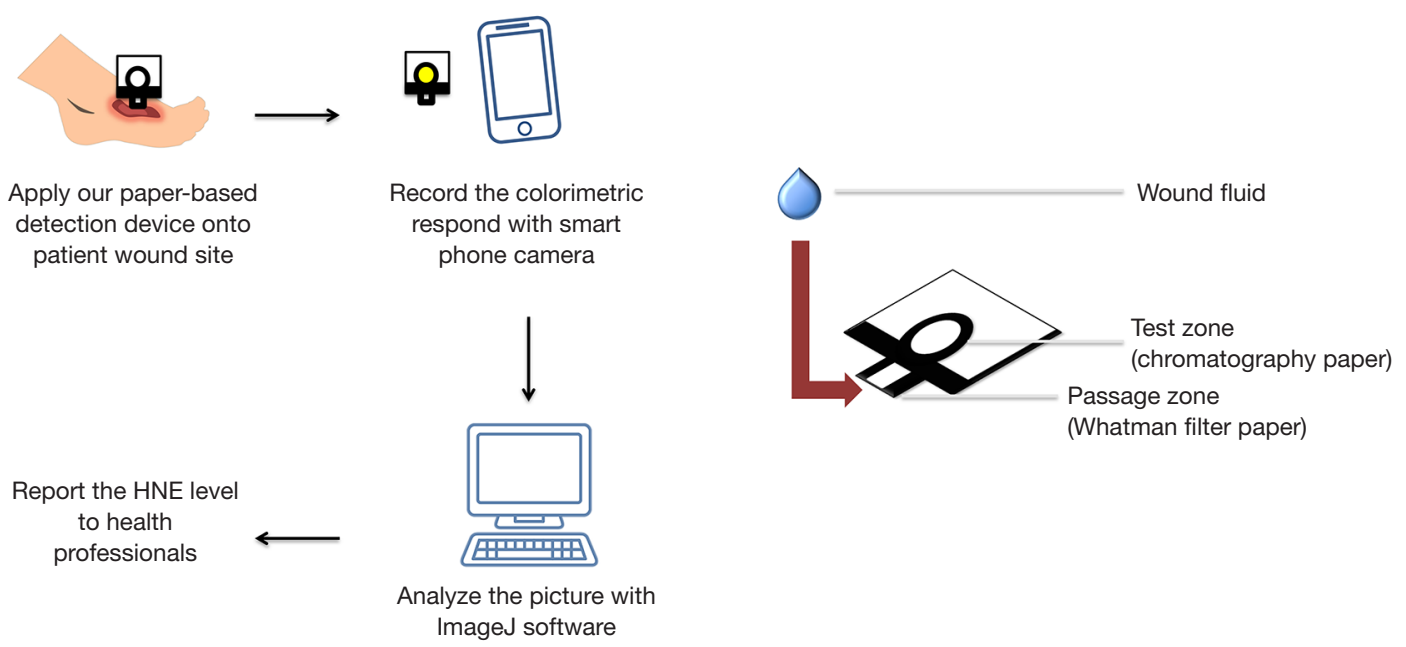

Figure 1 Workflow and two-dimensional schematic of our paper-based detection device.

and death (3).

Due to the decreased healing rate and the increased prevalence of comorbid conditions among the elderly (i.e., diabetes, cardiovascular diseases, cancer, and strokes, which may impede wound healing), a significantly higher incidence of chronic wounds was found in the geriatric population compared to younger populations (5). Other adverse events caused by increased age, such as lack of energy, neurodegeneration, and changes in skin architecture, may also lead to higher risk for developing chronic wounds (5). Furthermore, elderly patients have a higher incidence for admission to hospitals due to wound complications. To avoid these complications, there is an urgent need for a simple, rapid, and effective point-of-care (POC) diagnostic tool for early wound assessment. Besides the common HNE detection methods such as fluorescence assays or ELISA, several biosensors that offer better sensitivity as well as specificity were developed, such as the FRET-based HNE detector or an aptamer-based assay coupled with magnetic beads $(6,7)$. However, although these devices provide superior ability to detect HNE, these methods are often complex to operate and require sophisticated laboratory equipment, which may not be applicable for clinical usage. In this study, we developed a paper-based diagnostic device that, coupled with a smartphone camera, can help monitor the healing progress of chronic wounds. With its accuracy, user-friendliness, fast-response, and low-cost, such a device may serve as a valuable POC diagnostic tool for wound assessment, and can thus improve currently adverse situations for the elderly in long-term care.

\section{Methods}

Our paper-based detection device was built to assay Human Neutrophil Elastase (HNE), one of the crucial serine proteases that modulates inflammation levels in chronic wounds, and used N-Methoxysuccinyl-Ala-Ala-Pro-Val p-nitroanilide as a chromogenic substrate (8). Studies have shown that HNE plays a vital role in the remodeling phase of wound healing. For example, an elevated HNE level may lead to excessive degradation of extracellular matrix as well as fibronectin (9-13). Compared to acute wounds, a higher level of HNE is also reported in chronic wounds, suggesting that HNE levels can serve as an indicator for wound assessment (14). The paper-based device we developed comprises two main parts:

(I) A test zone (chromatography paper FN5); and (II) a passage zone (Whatman filter paper No. 1). We created hydrophobic barrier test zones in our chromatography paper using wax printing, and fabricated our device by combining our test zone and passage zone portions into a single device (Figure 1). We selected chromatography paper for our test zone and Whatman filter paper for our passage zone in order to combine and leverage the positive characteristics of both paper types. This allowed us to create a device that worked rapidly and provided a superior colorimetric signal. More details, including fabrication and rationale, can be found in our previously published study (15).

To further demonstrate our detection device, we measured the level of HNE via the following steps: (I) immobilization of N-Methoxysuccinyl-Ala-Ala-Pro-Val 


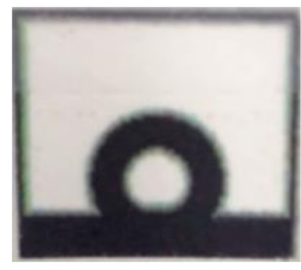

Before reaction

Colorless

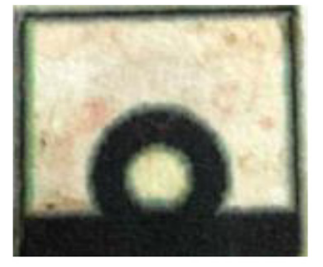

After reaction

Light yellow
Figure 2 The colorimetric results from our paper-based detection device in clinical use. The color change (from colorless to light yellow) can be observed with the naked eye.

p-nitroanilide onto the test zone; (II) immobilization of $3 \%$ BSA onto the passage zone to prevent non-specific binding; (III) incubation of both test zone and passage zone at $32{ }^{\circ} \mathrm{C}$ to dry out the device; (IV) combination of two pieces into one device using adhesive tape; (V) absorption of wound fluid by placing our device onto patient wound; (VI) recording of the color change with a smartphone; and (VII) analysis of the colorimetric results and conversion of the results into HNE levels using ImageJ software.

\section{Results}

The sensitivity of our paper-based detection device was previously tested using both buffer and tear systems, and the limit of detection (LOD) for both systems was approximately 0.0186 and $0.76 \mu \mathrm{g} / \mathrm{mL}$, respectively (15). To evaluate the real-world applicability of our paper-based detection device, we tested it by directly applying it to patient wounds. Figure 2 shows that our device provided a visible colorimetric response (from colorless to light yellow) after absorbing wound fluid from patient 4 (Figure 3), indicating that our device can be successfully applied in a clinical setting without requiring any expensive analytical equipment or complex operating methods. In order to quantify the HNE level from each patient, we converted the recorded colorimetric response to blue using ImageJ software and defined our mean intensity as (test zone intensity) - (blank zone intensity). A total of 8 patients were examined, and the HNE mean values were calculated as presented in Table 1. According to the results we conducted and other previously published research, a higher level of HNE can be found in delayed healing wounds (13-15). In this study, high levels of HNE were recorded from all 8 chronic wound patients, suggesting a retarded wound

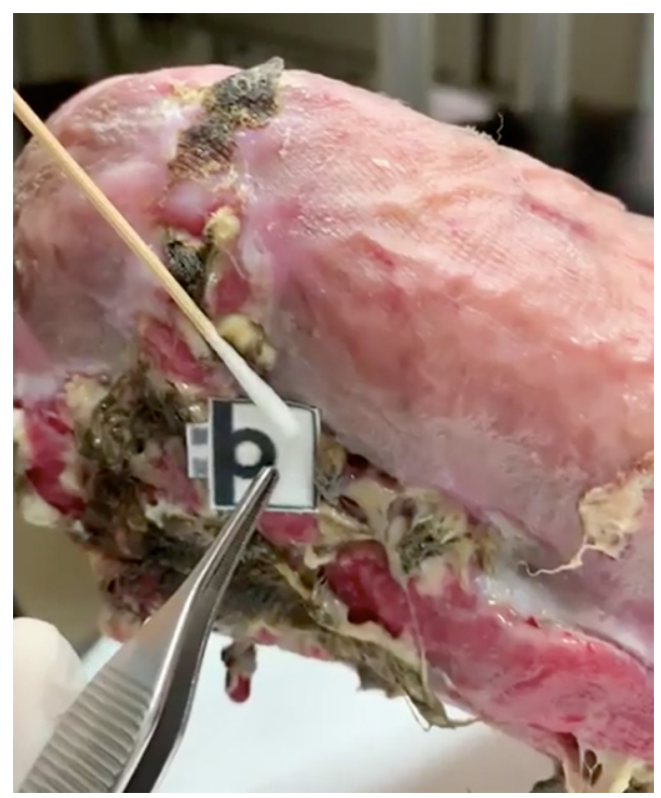

Figure 3 Demonstration of our paper-based detection device on an operating table.

Table 1 Mean intensities of HNE between different patients as detected by our paper-based device

\begin{tabular}{lcccc}
\hline Sample & Sex & Age & Wound type & $\begin{array}{c}\text { HNE mean } \\
\text { intensity (a.u.) }\end{array}$ \\
\hline 1 & M & 79 & Chronic wound & 107.2 \\
2 & M & 56 & Chronic wound & 120.5 \\
3 & M & 68 & Chronic wound & 70.5 \\
4 & F & 62 & Chronic wound & 92.5 \\
5 & M & 58 & Chronic wound & 43.5 \\
6 & F & 59 & Chronic wound & 150.5 \\
7 & F & 52 & Chronic wound & 55.5 \\
8 & F & 53 & Chronic wound & 60.5 \\
\hline
\end{tabular}

HNE, human neutrophil elastase.

healing status that is consistent with our earlier study.

\section{Discussion}

Paper-based materials have been widely used for various diagnostic devices for clinical tests using a variety of sample sources including blood, urine, saliva, and even food (16-19). Moreover, due to their low-cost, convenience, and userfriendliness, these diagnostic tools can be applied in 
equipment-, resource-, and energy source-poor areas and can also benefit disabled patients that have difficulty reaching medical resources.

Several methods for HNE detection have been developed including the use of chromogenic substrates, ELISA, and chemical probes $(20,21)$. Although some of them offer superior sensitivity or can be operated under complex systems, they require complicated analytical equipment and are difficult to use for disabled patients. Currently, there is no POC device that can semi-quantify the amount of HNE level for chronic wound monitoring.

Compared to current HNE detection methods, our paper-based HNE detection device comprises the following advantages: (I) fast response-our device needs only 15 minutes to complete its colorimetric reaction; (II) a small sample volume requirement-only $3 \mu \mathrm{L}$ of patient sample is required for a test; (III) good sensitivity - our device can detect $\mu \mathrm{g} / \mathrm{mL}$ levels of HNE in wound fluid system; and (IV) user-friendliness-as shown in Figure 3, this device can be operated without sophisticated laboratory facilities and can be easily performed. For these reasons, our detection device may improve wound assessment and care in elderly healthcare centers as well as in long-term care situations in the home.

To better position our device for clinical use, several issues must still be addressed. First of all, due to the lack of any standard wound fluid system for us to reference, we could only calculate each patient's HNE level by establishing his or her individual calibration curve beforehand. In the near future, we hope to combine our device with another smartphone-based application that can calculate HNE level in a faster and even more convenient manner. Secondly, although the pNA substrate we used is relative easy to obtain, there is a risk of cross-reaction during diagnosis. The complex wound environment contains various enzymes or proteins that can also react with our substrate, resulting in a higher HNE readout. Furthermore, when placing our device directly onto patient wounds, other interference such as bloody substances may influence our colorimetric results. To solve these problems, we will search for other substrates that have higher specificity to HNE and provide different color changes that can be more easily distinguished from bloody fluids.

The development of a rapid, inexpensive, easy-to-use, and reliable diagnostic tool for chronic wound care will benefit not only patients but also their family members and the healthcare community as a whole. By applying our device, we hope to provide early chronic wound detection, decrease medical costs, and reduce older patients' clinical visit time as well as the possible risks involved with transportation to a possibly distant clinical setting.

\section{Acknowledgments}

Funding: This study is funded by project of 107-2628-E-007-001-MY3 to CMC; 108-2314-B-006-080MY3 to SCP, from the Ministry of Science and Technology, Taiwan.

\section{Footnote}

Data Sharing Statement: Available at http://dx.doi. org/10.21037/ht-20-6

Peer Review File: Available at http://dx.doi.org/10.21037/ht20-6

Conflicts of Interest: All authors have completed the ICMJE uniform disclosure form (available at http://dx.doi. org/10.21037/ht-20-6). The authors have no conflicts of interest to declare.

Ethical Statement: The authors are accountable for all aspects of the work in ensuring that questions related to the accuracy or integrity of any part of the work are appropriately investigated and resolved. In this study, ethics committee approval and informed consent were not considered to be necessary.

Open Access Statement: This is an Open Access article distributed in accordance with the Creative Commons Attribution-NonCommercial-NoDerivs 4.0 International License (CC BY-NC-ND 4.0), which permits the noncommercial replication and distribution of the article with the strict proviso that no changes or edits are made and the original work is properly cited (including links to both the formal publication through the relevant DOI and the license). See: https://creativecommons.org/licenses/by-nc-nd/4.0/.

\section{References}

1. Lazarus GS, Cooper DM, Knighton DR, et al. Definitions and guidelines for assessment of wounds and evaluation of healing. Wound Repair Regen 1994;2:165-70.

2. Werdin F, Tennenhaus M, Schaller HE, et al. Evidencebased management strategies for treatment of chronic 
wounds. Eplasty 2009;9:e19.

3. Järbrink K, Ni G, Sönnergren H, et al. The humanistic and economic burden of chronic wounds: a protocol for a systematic review. Syst Rev 2017;6:15.

4. Walshe C. Living with a venous leg ulcer: a descriptive study of patients' experiences. J Adv Nurs 1995;22:1092-100.

5. Gould LJ. Wound healing in older adults. R I Med J (2013) 2016;99:34.

6. Schulenburg C, Faccio G, Jankowska D, et al. A FRETbased biosensor for the detection of neutrophil elastase. Analyst 2016;141:1645-8.

7. Zhao Q, Li XF, Le XC. Aptamer capturing of enzymes on magnetic beads to enhance assay specificity and sensitivity. Anal Chem 2011;83:9234-36.

8. Palolahti M, Lauharanta J, Stephens RW, et al. Proteolytic activity in leg ulcer exudate. Exp Dermatol 1993;2:29-37.

9. Yager DR, Nwomeh BC. The proteolytic environment of chronic wounds. Wound Repair Regen 1999;7:433-41.

10. Henson PM, Johnston R. Tissue injury in inflammation. Oxidants, proteinases, and cationic proteins. J Clin Invest 1987;79:669-74.

11. Grinnell F, Zhu M. Fibronectin degradation in chronic wounds depends on the relative levels of elastase, $\alpha 1$ proteinase inhibitor, and $\alpha 2$-macroglobulin. J Invest Dermatol 1996;106:335-41.

12. Rao CN, Ladin DA, Chilukuri K, et al. $\alpha 1$-antitrypsin is degraded and non-functional in chronic wounds but intact and functional in acute wounds: the inhibitor protects fibronectin from degradation by chronic wound fluid enzymes. J Invest Dermatol 1995;105:572-8.

doi: 10.21037/ht-20-6

Cite this article as: Yang T, Pan SC, Cheng CM. Paperbased detection device for chronic wound monitoring. Health Technol 2020;4:5.
13. Schultz GS, Ladwig G, Wysocki A. Extracellular matrix: review of its roles in acute and chronic wounds. World Wide Wounds 2005;2005:1-18.

14. Bai Y, Wang H, Zhao Q. Detection of human neutrophil elastase by aptamer affinity capillary electrophoresis coupled with laser-induced fluorescence using specified site fluorescently labeled aptamer. Anal Bioanal Chem 2017;409:6843-9.

15. Yang T, Pan SC, Cheng CM. Paper-based human neutrophil elastase detection device for clinical wound monitoring. Lab Chip 2020;20:2709-16.

16. Jokerst JC, Adkins JA, Bisha B, et al. Development of a paper-based analytical device for colorimetric detection of select foodborne pathogens. Anal Chem 2012;84:2900-7.

17. Cheng CM, Martinez AW, Gong J, et al. Paper-based ELISA. Angew Chem Int Ed Engl 2010;49:4771-4.

18. Lin SC, Tzeng CY, Lai PL, et al. Paper-based CRP monitoring devices. Sci Rep 2016;6:38171.

19. Sechi D, Greer B, Johnson J, et al. Three-dimensional paper-based microfluidic device for assays of protein and glucose in urine. Anal Chem 2013;85:10733-7.

20. Ferreira AV, Antunes E, Ribeiro A, et al. Design of a chromogenic substrate for elastase based on split GFP system-Proof of concept for colour switch sensors. Biotechnol Rep (Amst) 2019;22:e00324.

21. Schulz-Fincke AC, Tikhomirov AS, Braune A, et al. Design of an Activity-Based Probe for Human Neutrophil Elastase: Implementation of the Lossen Rearrangement To Induce Forster Resonance Energy Transfers. Biochemistry 2018;57:742-52. 